Research Article

\title{
Feedback Linearization Control for Highly Uncertain Nonlinear Systems Augmented by Single-Hidden-Layer Neural Networks
}

\author{
Hamou Ait Abbas ${ }^{*}$, , Mohammed Belkheiri ${ }^{2}$ and Boubakeur Zegnini ${ }^{1}$ \\ ${ }^{1}$ Laboratoire d'Etude et de Développement des Matériaux Semi Conducteurs et Diélectriques \\ ${ }^{2}$ Laboratoire de Télécommunications, Signaux et Systèmes. \\ Université Amar Telidji- Laghouat, BP G37 Route de Ghardaïa (03000 Laghouat), Algérie.
}

Received 30 September 2014; Revised 07 November 2014; Accepted 27 November 2014

\begin{abstract}
The main objective of this paper is to design an adaptive output feedback control for a class of uncertain nonlinear systems using only one Single-Hidden-Layer (SHL) Neural Networks (NN) in order to eliminate the unstructured uncertainties. The approach employs feedback linearization, coupled with an on-line $\mathrm{NN}$ to compensate for modelling errors. A fixed structure dynamic compensator is designed to stabilize the linearized system. A signal, comprised of a linear combination of the measured tracking error and the compensator states, is used to adapt the NN weights. The network weight adaptation rule is derived from Lyapunov stability analysis, and guarantees that the adapted weight errors and the tracking error are bounded. Numerical simulations of both nonlinear systems, Van der Pol example and tunnel diode circuit model, having full relative degree, are used to illustrate the practical potential of the proposed approach.
\end{abstract}

Keywords: Feedback control, nonlinear systems, single-hidden-layer neural network, error dynamics, unstructured uncertainty.

\section{Introduction}

Nonlinearities exist in a wide range of physical systems and devices, such as chaotic systems, electromagnetism, mechanical actuators, electronic relay circuits and other areas. Unfortunately, such nonlinearity often limits system performance. A fundamental ambition for many research efforts in control theory is the development of a powerful control for highly uncertain nonlinear systems, to name a few, that are poorly modelled and influenced by the parameter variations.

In recent decades, adaptive control design has been significantly advanced for nonlinear systems, e.g., Brunovsky systems $[3,9,15]$, feedback-linearized systems [2,25,30], and strict-feedback systems [32,35,38], and various controllers have been proposed using different techniques, namely, the sliding-mode control or variable structure control [26,31], adaptive backstepping control $[13,36]$, and exact state feedback linearization control $[5,12]$. The three approaches have their own merits whereas with some defects. For example, the sliding-mode control system is immune to parameters variation and disturbances while it

* E-mail address: h.aitabbas@lagh-univ.dz ISSN: 1791-2377 @ 2015 Kavala Institute of Technology. All rights reserved.

has chattering problem. The adaptive backstepping control specifically applied for triangular systems can deal with matched or unmatched parameter uncertainties whereas it needs more calculation and longer settling time. The exact state feedback linearization control is among the most successful techniques to achieve input/output decoupling and high dynamic performance but sensitive to parameter variations so any mismatch could cause system instability.

The main drawback of such methods is that they rely on an exact model of the systems to be controlled with precise parameters. Taking advantage of this fact, adaptive output feedback control is expected to exhibit more excellent performance, because it requires little knowledge about system mathematical model and parameters. Consequently, it has been recognized as a powerful methodology for designing feedback controllers for highly uncertain nonlinear systems with parametric uncertainties. Different adaptive state feedback and output feedback control algorithms have been developed for a reasonably large class of nonlinear systems under various uncertain nonlinearities(e.g., see [16,17,35]). Thus, output feedback control of nonlinear uncertain systems, using a high-gain observer, was introduced in [1] and [11]. A solution to the output feedback stabilization problem for systems in which nonlinearities depend only upon the available measurement was given in [28]. In [20] and [22], we find backsteppingbased approaches to adaptive output feedback control of uncertain systems that are linear with respect to unknown parameters. Hence, development of an alternative approach 
to treat the intelligent tracking control of uncertain nonlinear systems is highly desirable.

Both neural network systems [4,5,35] and fuzzy logic systems $[26,31,38]$ have been successfully applied in recent years for several engineering applications to universally approximate the unstructured uncertainties of dynamic systems. Many important adaptive neural-network-based and fuzzy-based control schemes have been developed to treat the robust control of uncertain nonlinear systems and various stable performance criteria are guaranteed by theoretical analyses $[8,17,18,33,37]$. A key assumption in most of these methods is that all the states of the plant are available for feedback. Recently, observer-based adaptive fuzzy-neural control schemes $[23,34]$ are proposed for a certain class of unknown nonlinear dynamical systems. Adaptive neural network output feedback schemes with high-gain observer $[16,29]$ are developed for the tracking control of a general class of nonlinear systems. Another method that involves design of an adaptive observer using function approximators and backstepping control can be found in $[5,10]$. In [2] and [19], adaptive state and output feedback neurocontrol is developed for full relative degree nonlinear systems.

The contribution of the present correspondence is to address the problem of designing an intelligent adaptive output feedback tracking controller for a class of uncertain nonlinear systems for which the output has full relative degree. The developed control scheme is based on an adaptive neural network system that is employed to for the modelling errors, and a linear error observer that is used to estimate the derivative of output. Using an approximate feedback linearizing control law, the nonlinear dynamics are inverted and the obtained tracking-error dynamics are almost linear. Taking advantage of this fact, we propose a linear observer for the almost linear error dynamics. The estimates provided by this observer are used as teaching signals to the NN. Ultimate boundedness is shown through Lyapunov's direct method. The theoretical results are illustrated in the design of a controller for both nonlinear systems having full relative degree, tunnel diode circuit (TDC) model and Van Der Pol (VDP) example.

The rest of this correspondence is organized as follows: In Section (2), the tracking control problem is formulated. Section (3) proposes a novel Output Feedback controller design applied to control the uncertain nonlinear system. Section (4) develops the controller design. NN augmentation is detailed in section (5). In Section (6), the rigorous stability analysis is presented to guarantee the boundedness of the tracking error elements. In Section (7), a simulation example is made. The last section is devoted to some concluding remarks.

\section{Problem Statement}

Consider the dynamics of an observable and stabilizable nonlinear SISO system given by the following equations:

$$
\begin{aligned}
& \dot{x}=f(x, u) \\
& y=h(x)
\end{aligned}
$$

where $x \in \Re^{n}$ is the state of the plant, $u \in \mathfrak{R}$, and $y \in \Re$ are the input (control) and output (measurement) signals, respectively.
Assumption.1: The functions $f: \mathfrak{R}^{n+1} \rightarrow \mathfrak{R}^{n} \quad$ and $h: \Re^{n} \rightarrow \mathfrak{R}$ are sufficiently smooth partially known, and the output has full relative degree $r$ for all $(x, u) \in \Omega \times \mathfrak{R}$ where $\Omega \subset \Re^{n}$.

Then following [29], there exists a mapping that transforms the system in (1) into the so-called normal form:

$\left\{\begin{array}{l}\dot{\xi}_{i}=\xi_{i+1}, \quad i=1, \ldots, r-1 \\ \dot{\xi}_{r}=h(\xi, u) \\ \xi_{1}=y\end{array}\right.$

where $h(\xi, u)=L_{f}^{(r)} \cdot h$ are the Lie derivatives, and $\xi=\left[\begin{array}{lll}\xi_{1} & \ldots & \xi_{r}\end{array}\right]^{T}$.

The objective is to synthesize a feedback control law that utilizes the available measurement $y$ so that $y(t)$ tracks a smooth bounded reference trajectory $y_{c}(t)$ with bounded error.

\section{Controller Design}

\subsection{Feedback Linearization}

Feedback linearization is approximated by defining the following control input signal:

$u=\hat{h}^{-1}(y, v)$

where $v$ is defined as:

$$
v=\hat{h}(y, u)
$$

and is commonly referred to as a pseudocontrol. The function $\hat{h}(y, u)$ represents the best available approximation of $h(y, u)$. Then, the system dynamics can be expressed as:

$y^{(r)}=v+\Delta$

where

$$
\Delta(\xi, v)=h\left(\xi_{1}, \hat{h}^{-1}\left(\xi_{1}, v\right)\right)-\hat{h}\left(\xi_{1}, \hat{h}^{-1}\left(\xi_{1}, v\right)\right)
$$

is the inversion error. The pseudocontrol is chosen to have the form:

$$
v=y_{c}^{(r)}+u_{d}^{c}-u_{a d}
$$

where $y_{c}^{(r)}$ is the $r^{\text {th }}$ derivative of the input signal, generated by a stable command filter, $u_{d}^{c}$ is the output of a linear dynamic compensator, $u_{a d}$ is the adaptive control signal designed to cancel $\Delta$.

With (7), the dynamics in (5) reduce to

$$
y^{(r)}=y_{c}^{(r)}+u_{d}^{c}-u_{a d}+\Delta
$$


From (6), notice that $\Delta$ depends on $u_{a d}$ through $v$. We will design $u_{a d}$ to approximately cancel $\Delta$. Therefore, the following assumption is introduced to guarantee the existence and uniqueness of a solution for $u_{a d}$ [27].

Assumption.2. The mapping $u_{a d} \rightarrow \Delta$ is a contraction over the entire input domain of interest.

A contraction is defined by the condition: $\left|\partial \Delta / \partial u_{a d}\right|<1$.

Using (6), this reduces to

$$
\left|\frac{\partial \Delta}{\partial u_{a d}}\right|=\left|\frac{\partial(h-\hat{h})}{\partial u} \frac{\partial u}{\partial v} \frac{\partial v}{\partial u_{a d}}\right|=\left|\frac{\partial h / \partial u}{\partial \hat{h} / \partial u}-1\right|<1
$$

Condition (9) is equivalent to the following requirements on $\hat{h}$ :

$$
\begin{aligned}
& \text { 1) } \operatorname{sgn}\left(\frac{\partial \hat{h}}{\partial u}\right)=\operatorname{sgn}\left(\frac{\partial h}{\partial u}\right) ; \\
& \text { 2) } \infty>\left|\frac{\partial \hat{h}}{\partial u}\right|>\frac{\left(\frac{\partial h}{\partial u}\right)}{2}>0 .
\end{aligned}
$$

where the first condition means that control reversal is not allowed, and the second condition places a lower bound on the estimate of the control effectiveness in (4).

\subsection{Dynamic Compensator Design}

Define the output tracking error as $\tilde{y}=y_{c}-y$. Then the dynamics in (8) can be rewritten as:

$$
\tilde{y}^{(r)}=-u_{d}^{c}+u_{a d}-\Delta
$$

For the case $\Delta=0$, the adaptive term $u_{a d}$ is not required and the error dynamics in (10) reduce to

$$
\tilde{y}^{(r)}=-u_{d}^{c}
$$

The following linear compensator is introduced to stabilize the dynamics in (11):

$$
\left\{\begin{array}{l}
\dot{\eta}=-A \eta+b_{c} \tilde{y} \\
u_{d}^{c}=c_{c} \eta+d_{c} \tilde{y}
\end{array} \quad \text { where } \eta \in \Re^{r-1}\right.
$$

Note that $\eta$ needs to be at least of dimension $(r-1)$ [6]. This follows from the fact that (11) corresponds to error dynamics that have $r$ poles at the origin. One could elect to design a compensator of dimension $\geq r$ as well. In the future, we will assume that the minimum dimension is chosen.

\subsection{Tracking Error Signal Analysis}

Returning to (10), notice that the vector $e=\left[\begin{array}{cccc}\tilde{y} & \dot{\tilde{y}} & \ldots & \tilde{y}^{(r-1)}\end{array}\right]^{T}$ mutually with the compensator state $\eta$ will obey the following dynamics, referred to as tracking error dynamics:

$\left\{\begin{array}{l}\dot{E}=\bar{A} E+\bar{b}\left[u_{a d}-\Delta\right] \\ z=\bar{C} E\end{array}\right.$

where $z$ is the vector of available measurements.

Reminder that:

$$
\begin{aligned}
E & =\left[\begin{array}{ll}
e^{T} & \eta^{T}
\end{array}\right]^{T}, \quad \bar{b}=\left[\begin{array}{ll}
b^{T} & 0
\end{array}\right]^{T} \\
\bar{A} & =\left[\begin{array}{cc}
A-d_{c} b c & -b c_{c} \\
b_{c} c & A_{c}
\end{array}\right] ; \quad \bar{c}=\left[\begin{array}{ll}
c & 0 \\
0 & I
\end{array}\right] ; \\
A & =\left(\begin{array}{ccccc}
0 & 1 & 0 & \ldots & 0 \\
0 & 0 & 1 & \ldots & 0 \\
\mathrm{M} & 0 & 0 & 0 & \mathrm{M} \\
0 & 0 & 0 & \ldots & 1 \\
0 & 0 & 0 & \ldots & 0
\end{array}\right) ; b=\left[\begin{array}{l}
0 \\
0 \\
\mathrm{M} \\
1
\end{array}\right] ; c=\left[\begin{array}{l}
1 \\
0 \\
\mathrm{M} \\
0
\end{array}\right]^{T}
\end{aligned}
$$

Noting that $A_{c}, b_{c}, c_{c}$ and $d_{c}$ in (12) should be designed such that $\bar{A}$ is Hurwitz.

\section{Design and Analysis of an Observer for the Error Dynamic}

For the full-state feedback application [7,14,21], Lyapunovlike stability analysis of the error dynamics in (13) results in update laws for the adaptive control parameters in terms of the error vector $E$. In [14,19,21], adaptive state observers are used to provide the necessary estimates in the adaptation laws. However, the stability analysis was limited to secondorder systems with position measurements. To rest these assumptions, we propose a simple linear observer for the tracking error dynamics in (13) and show through Lyapunov's direct method that the adaptive part of the control signal $\left(u_{a d}\right)$ can compensate for the inversion error $(\Delta)$, if the output of this observer is used as an error signal for the adaptive laws.

A minimal-order observer of dimension $(r-1)$ may be designed for the dynamics in (13). To this end, consider the following linear observer for the tracking error dynamics in:

$\left\{\begin{array}{l}\dot{\hat{E}}=\bar{A} \hat{E}+K(z-\hat{z}) \\ \hat{z}=\bar{C} \hat{E}\end{array}\right.$

where $K$ is a gain matrix, and should be chosen such that $(\bar{A}-K \bar{C})$ is asymptotically stable, and $z$ is defined in (13).

The following remark will be useful in the sequel. 
Theorem 1. Equation (15) provides estimates only for the states that are feedback linearizable and not for the states that are associated with the internal dynamics.

Let

$\tilde{A}=\bar{A}-K \bar{C}, \quad \tilde{E}=\hat{E}-E$

Then, the observer error dynamics can be written

$\dot{\tilde{E}}=\tilde{A} \tilde{E}-\bar{b}\left[u_{a d}-\Delta\right]$

\section{SHL NN Approximation of the Inversion Error}

\subsection{NN Approximation}

Given $x \in \mathfrak{R}^{N_{1}}$, an SHL NN has an output given by

$y_{i}=\sum_{j=1}^{N_{2}}\left[m_{i j} \sigma\left(\sum_{k=1}^{N_{1}} n_{j k} x_{k}+\theta_{n j}\right)+\theta_{m i}\right]$

$i=1, \ldots, N_{3}$

where $\sigma($.$) is activation function, n_{j k}$ are the first-to-second layer interconnection weights, $m_{i j}$ are the second to third layer interconnection weights, $N_{2}$ is associated with the number of neurons in the hidden layer, $\theta_{n j}$ and $\theta_{m i}$ and are bias terms.

The following theorem extends these results to map the unknown dynamics of an observable system from available input-output history [7].

Theorem 2: Given a compact set $D \subset R^{n+1}$ and $\in^{*}$, the model inversion error $\Delta(\xi, v)$ can be approximated over $D$ by an SHL NN

$$
\Delta(x, u)=M^{T} \sigma\left(\Psi^{T} \mu\right)+\in(d, \mu), \quad|\in|<\epsilon^{*}
$$

using the input vector

$$
\mu(t)=\left[\begin{array}{lll}
1 & \bar{v}_{d}{ }^{T}(t) & \bar{y}_{d}{ }^{T}(t)
\end{array}\right]^{T},\|\mu\| \leq \mu^{*}, \mu^{*}>0
$$

where

$$
\begin{aligned}
\bar{v}_{d}^{T}(t) & =\left[\begin{array}{llll}
v(t) & v(t-d) & \ldots & v\left(t-\left(n_{1}-r-1\right) d\right)
\end{array}\right]^{T} \\
\bar{y}_{d}{ }^{T}(t) & =\left[\begin{array}{llll}
y(t) & y(t-d) & \ldots & y\left(t-\left(n_{1}-r-1\right) d\right)
\end{array}\right]^{T}
\end{aligned}
$$

with $n_{1} \geq n, d>0$ denoting time-delay and $\mu^{*}$ being a uniform bound for all $(x, u) \in D$.

\subsection{Adaptive Control}

The adaptive signal is chosen to be the output of an SHL NN $u_{a d}=\hat{M}^{T} \sigma\left(\hat{\Psi}^{T} \mu\right)$

where $\hat{M}$ and $\hat{\Psi}$ are estimates of $M$ and $\Psi$ that are updated according to the following adaptation laws:

$\dot{\dot{\Psi}}=-G\left[2 \mu \hat{E}^{T} P \bar{b} \hat{M}^{T} \hat{\Psi}^{\prime}+k\left(\hat{\Psi}-\Psi_{0}\right)\right]$

$\dot{\hat{M}}=-F\left[2\left(\widehat{\sigma}-\hat{\sigma}^{\prime} \hat{\Psi}^{T} \mu\right) \hat{E}^{T} P \bar{b}+k\left(\hat{M}-M_{0}\right)\right]$

in which $M_{0}$ and $\Psi_{0}$ are initial values of the NN weights, $\hat{\sigma}=\sigma\left(\Psi_{0}^{T} \mu\right), \hat{\sigma}^{\prime}$ denotes the Jacobian matrix, $P$ is the solution of the Lyapunov equation

$\bar{A}^{T} P+P \bar{A}=-Q$

for some $Q>0, k>0$ and $F$ is adaptation gain matrices.

Notice that in (21), there is an algebraic loop, since $\mu$, by definition, depends upon $u_{a d}$ through $v$, see (20). However, with bounded squashing functions, this algebraic loop has at least one fixed-point solution.

Using (19) and (21), the error dynamics in (13) can be formulated as:

$\left\{\begin{array}{l}\dot{E}=\bar{A} E+\bar{b}\left[\hat{M}^{T} \sigma\left(\hat{\Psi}^{T} \mu\right)-M^{T} \sigma\left(\Psi^{T} \mu\right)-\epsilon\right] \\ z=\bar{C} E\end{array}\right.$

Define

$$
\tilde{M}=\hat{M}-M, \quad \tilde{\Psi}=\widehat{\Psi}-\Psi, \quad \tilde{Z}=\left[\begin{array}{cc}
\tilde{M} & 0 \\
0 & \tilde{\Psi}
\end{array}\right]
$$

and note that

$\|\hat{M}\|<\|\tilde{M}\|+M^{*}, \quad\|\hat{\Psi}\|_{F}<\|\tilde{\Psi}\|_{F}+\Psi^{*}$

where $M^{*}$ and $\Psi^{*}$ are the upper bounds for the weights in (19).

$\|\hat{M}\|<M^{*}, \quad\|\hat{\Psi}\|_{F}<\Psi^{*}$

The subscript $F$ denoting the Frobenius norm.

With (26), the representation

$u_{a d}-\Delta=\hat{M}^{T} \sigma\left(\hat{\Psi}^{T} \mu\right)-M^{T} \sigma\left(\Psi^{T} \mu\right)-\epsilon$

allows for the following upper bound for some computable $\alpha_{1}, \alpha_{2}$ :

$\left|u_{a d}-\Delta\right| \leq \alpha_{1}|| \tilde{Z} \|_{F}+\alpha_{2}, \quad \alpha_{1}>0, \quad \alpha_{2}>0$ 
For the stability proof, we will need the following representation:

$$
\begin{aligned}
\hat{M}^{T} \sigma\left(\hat{\Psi}^{T} \mu\right)-M^{T} \sigma\left(\Psi^{T} \mu\right)= & \tilde{M}^{T}\left(\hat{\sigma}-\hat{\sigma}^{\prime} \hat{\Psi}^{T} \mu\right) \\
& +\hat{M}^{T} \hat{\sigma}^{\prime} . \hat{\Psi}^{T} \mu+\chi
\end{aligned}
$$

where

$\chi=\tilde{M}^{T} \hat{\sigma}^{\prime} \cdot \Psi^{T} \mu-M^{T} \partial\left(\tilde{\Psi}^{T} \mu\right)^{2}$

Such a representation is achieved via Taylor series expansion of $\sigma\left(\Psi^{T} \mu\right)$ around the estimates $\hat{\Psi}^{T} \mu$ (refer to [24] for more details).

With the bound in (20), a bound for $(\chi-\epsilon)$ over a compact set can be presented as follows [24]:

$|x-\epsilon| \leq \gamma_{1} \mid\|\tilde{Z}\|_{F}+\gamma_{2}, \quad \gamma_{1}>0, \quad \gamma_{2}>0$

where $\gamma_{1}$ and $\gamma_{2}$ are computable constants, $\gamma_{1}$ depends upon unknown constant $\mu^{*}$, and $\gamma_{2}$ upon $\in^{*}$. Thus, the forcing term in (24) can be written as:

$$
u_{a d}-\Delta=\tilde{M}^{T}\left(\hat{\sigma}-\hat{\sigma}^{\prime} . \hat{\Psi}^{T} \mu\right)+M^{T} \hat{\sigma}^{\prime} \hat{\Psi}^{T} \mu+\chi-\epsilon
$$

\section{Stability Analysis}

In this section, we confirm through Lyapunov's direct method that if the initial errors of the variables $E^{T}, \tilde{E}^{T}, \tilde{M}$, and $\tilde{\Psi}$ belong to a prescribed compact set, then they are eventually bounded. We will denote the composite error vector

$\zeta=\left[\begin{array}{lll}E^{T} & \tilde{E}^{T} & \operatorname{vec} \tilde{Z}^{T}\end{array}\right]^{T}$

Recall that (19) introduces the compact set $D$ over which the $\mathrm{NN}$ approximation is valid. From (19), it follows that

$$
\bar{x}=\left[\begin{array}{ll}
x^{T} & u
\end{array}\right]^{T} \in D \Leftrightarrow \bar{\omega}=\left[\begin{array}{ll}
\xi^{T} & u
\end{array}\right]^{T} \in \Omega_{D}
$$

where $\Omega_{D}=\Phi(D), \Phi($.$) being introduced in (2). The set$ $\Omega_{D}$ can be viewed as $\Omega_{D}=\Omega_{\xi} \times \Omega_{u}$, where $\xi \in \Omega_{\xi}, u \in \Omega_{u}$ all being compact sets. Notice that the static map in (3) and (4) ensure that $v \in \Omega_{v}, \Omega_{v}$ being a compact set. The relationship in (7) and the boundedness of $u_{d}^{c}$, as a stabilizer of (11), ensure that $\eta \in \Omega_{\eta}$, and $\hat{Z} \in \Omega_{\hat{Z}}$, these two sets being compact as well. Also notice that since the observer in (15) is driven by the output tracking error $\tilde{y}=y_{c}-y$ and compensator states $\eta$, having $\xi \in \Omega_{\xi}, y_{c} \in \Omega_{y_{c}}$, $\eta \in \Omega_{\eta}$, implies that $\hat{E} \in \Omega_{\hat{E}}$, the latter being a compact set.
The vector $\zeta$ in (32) can be viewed as a function of the state variables $\xi, \eta, \hat{E}, \hat{Z}$, the command vector $y_{c}=\left[\begin{array}{llll}y_{c} & \dot{y}_{c} & \cdots & y_{c}^{(r-1)}\end{array}\right]^{T}$ and the constant matrix $Z$

$\zeta=F\left(\xi, y_{c}, \eta, \hat{E}, \hat{Z}, Z\right)$

where $\hat{Z}=\left[\begin{array}{cc}\hat{M} & 0 \\ 0 & \hat{\Psi}\end{array}\right]$ and $Z=\left[\begin{array}{cc}M & 0 \\ 0 & \Psi\end{array}\right]$.

The relation in (34) represents a mapping from the original domains of the arguments to the space of the error variable

$$
F: \Omega_{\xi} \times \Omega_{y_{c}} \times \Omega_{\eta} \times \Omega_{\hat{E}} \times \Omega_{\hat{Z}} \times \Omega_{Z} \rightarrow \Omega_{\zeta}
$$

Thus, (2), (33), and the implicit dependence in (34) ensure that $\Omega_{\zeta}$ is a bound set. Introduce the largest ball, which is included in $\Omega_{\zeta}$ in the error space

$B_{R}=\{|\zeta| \cdot\|\zeta\| \leq R\}, R>0$

For every $\zeta \in B_{R}$, we have $\bar{x} \in D$ where $D$ is the set over which the $\mathrm{NN}$ approximation has been defined.

Assumption .3. Assume

$R>\gamma \sqrt{\frac{\Gamma_{M}}{\Gamma_{m}}} \geq \gamma$

where $\Gamma_{M}$ and $\Gamma_{m}$ are the maximum and minimum eigenvalues of the following matrix:

$\Gamma=\frac{1}{2}\left[\begin{array}{cccc}2 P & 0 & 0 & 0 \\ 0 & 2 \tilde{P} & 0 & 0 \\ 0 & 0 & F^{-1} & 0 \\ 0 & 0 & 0 & G^{-1}\end{array}\right]$

and $\gamma$ is defined as: $\gamma=\max \left(C_{1}, C_{2}, C_{3}\right)$.

Noting that

$$
\begin{aligned}
& C_{1}=\sqrt{\frac{\|P \bar{b}\|^{2} \gamma_{2}^{2}+k_{2}^{2}+\bar{Z}}{\lambda_{\text {min }}(Q)-2}}, \\
& C_{2}=\sqrt{\frac{\|P \bar{b}\|^{2} \gamma_{2}^{2}+k_{2}^{2}+\bar{Z}}{\lambda_{\text {min }}(\tilde{Q})-2}}, \\
& C_{3}=\sqrt{\frac{\|P \bar{b}\|^{2} \gamma_{2}^{2}+k_{2}^{2}+\bar{Z}}{\frac{k}{2}-k_{1}^{2}-\left[\gamma_{1}\|P \bar{b}\|\right]^{2}}},
\end{aligned}
$$


where

$$
\begin{aligned}
& \bar{Z}=\frac{k}{2}\left[\left\|M-M_{0}\right\|+\left\|\Psi-\Psi_{0}\right\|_{F}^{2}\right], k>2\left[k_{1}^{2}+\gamma_{1}^{2}\|P \bar{b}\|^{2}\right], \\
& k_{1}=\Theta \alpha_{1}+\|P \bar{b}\| \gamma_{1}, k_{2}=\Theta \alpha_{2}+\|P \bar{b}\| \gamma_{2}, \Theta=\|P \bar{b}\|+\|\tilde{P} \bar{b}\|
\end{aligned}
$$

and $\tilde{P}$ satisfies $\tilde{A}^{T} \tilde{P}+\tilde{P} \tilde{A}=-\tilde{Q}$ for some $\tilde{Q}>0$ with minimum eigenvalues $\lambda_{\text {min }}(\tilde{Q})>2$.

Assumption 3 may be interpreted as implying both an upper and lower bound for the adaptation gains. Define $\bar{\gamma}=\max \left(\lambda_{\max }(F), \lambda_{\max }(G)\right), \underline{\gamma}=\min \left(\lambda_{\min }(F), \lambda_{\min }(G)\right), \quad$ and $\bar{\lambda}=\max \left(\lambda_{\max }(P), \lambda_{\max }(\tilde{P})\right)$, and $\underline{\lambda}=\min \left(\lambda_{\min }(P), \lambda_{\min }(\tilde{P})\right)$, where $\lambda($.$) denotes the eigenvalue.$

Then, an upper bound for the adaptation gains results when $2 \bar{\lambda} \gamma>1$ and $2 \underline{\gamma} \bar{\gamma}>1$ for which the relation in (37) reduces to $\bar{\gamma}<R^{2} /\left(2 \gamma^{2} \bar{\lambda}\right)$. A lower bound for the adaptation gains when $2 \bar{\lambda} \gamma<1$ and $2 \underline{\lambda} \bar{\gamma}<1$, for which (37) reduces to $\underline{\gamma}>\gamma^{2} /\left(2 \bar{R}^{2} \underline{\lambda}\right)$.

Theorem 3: Let the assumptions 1-3 hold, and let $\lambda_{\text {min }}(Q)>2$ for $Q$ introduced in (23). Then, if the initial errors belong to the compact set $\Omega_{\alpha}$, defined in (49), the feedback control law given by (3) and (7), along with (22), guarantees that the signals $E, \tilde{E}, \tilde{M}$ and $\tilde{\Psi}$ in the closedloop system are ultimately bounded.

Proof: Consider the following Lyapunov function for the system in (17) and (24):

$$
V=E^{T} P E+\tilde{E}^{T} \tilde{P} \tilde{E}+\frac{1}{2} \tilde{M}^{T} F^{-1} \tilde{M}+\frac{1}{2} \operatorname{tr}\left(\tilde{\Psi}^{T} G^{-1} \tilde{\Psi}\right)
$$

The derivative of along (17) and (24) will be

$$
\begin{aligned}
\dot{V}= & -E^{T} P E-\tilde{E}^{T} \tilde{Q} \tilde{E}+2 E^{T} P \bar{b}\left[u_{a d}-\Delta\right]-2 \tilde{E}^{T} \tilde{P} \bar{b}\left[u_{a d}-\Delta\right] \\
& +\tilde{M}^{T} F^{-1} \dot{\tilde{M}}+\operatorname{tr}\left(\tilde{\Psi}^{T} G^{-1} \dot{\tilde{\Psi}}\right) .
\end{aligned}
$$

With the definition of $\tilde{E}=\hat{E}-E$ and (31), this can be written as:

$$
\begin{aligned}
\dot{V}= & -E^{T} P E-\tilde{E}^{T} \tilde{Q} \tilde{E}+2 E^{T} P \bar{b}\left[v_{a d}-\Delta\right]-2 \tilde{E}^{T} \tilde{P} \bar{b}\left[u_{a d}-\Delta\right] \\
& +2 \hat{E}^{T} \tilde{P} \bar{b}\left[\tilde{M}^{T}\left(\hat{\sigma}-\hat{\sigma}^{\prime} \cdot \hat{\Psi}^{T} \mu\right)+\hat{M}^{T} \hat{\sigma}^{\prime} \hat{\Psi}^{T} \mu+\chi-\epsilon\right] \\
& -2 \tilde{E}^{T}(\tilde{P} \bar{b}+P \bar{b})\left[u_{a d}-\Delta\right]+\tilde{M}^{T} F^{-1} \dot{\tilde{M}}+\operatorname{tr}\left(\tilde{\Psi}^{T} G^{-1} \dot{\tilde{\Psi}}\right)
\end{aligned}
$$

Substituting the adaptive laws implies

$$
\begin{aligned}
\dot{V}= & -E^{T} P E-\tilde{E}^{T} \tilde{Q} \tilde{E}+2 \hat{E}^{T} P \bar{b}[\chi-\epsilon] \\
& -2 \tilde{E}^{T}(\tilde{P} \bar{b}+P \bar{b})\left[u_{a d}-\Delta\right] \\
& -k \tilde{M}^{T} F^{-1}\left(\hat{M}-M_{0}\right)-k \cdot \operatorname{tr}\left[\tilde{\Psi}^{T}\left(\hat{\Psi}-\Psi_{0}\right)\right] .
\end{aligned}
$$

Using upper bounds from (29) and (30), the derivative of the Lyapunov function candidate can be upper bounded as:

$$
\begin{aligned}
\dot{V} \leq & -\lambda_{\text {min }}(Q)\|E\|^{2}-\lambda_{\text {min }}(\tilde{Q})\|\tilde{E}\|^{2} \\
& +2\|P \bar{b}\|\|\hat{E}\|\left[\gamma_{1}|| \tilde{Z} \|_{F}+\gamma_{2}\right]+2 \Theta\|\tilde{E}\|\left[\alpha_{1}\|\tilde{Z}\|_{F}+\alpha_{2}\right] \\
& -\frac{k}{2}\|\tilde{M}\|^{2}-\frac{k}{2}\left\|\hat{M}-M_{0}\right\|^{2}+\frac{k}{2}\left\|M-M_{0}\right\|^{2} \\
& -\frac{k}{2}\|\tilde{\Psi}\|_{F}^{2}-\frac{k}{2}\left\|\hat{\Psi}-\Psi_{0}\right\|_{F}^{2}+\frac{k}{2}\left\|\Psi-\Psi_{0}\right\|_{F}^{2}
\end{aligned}
$$

where the following property for matrices has been used:

$$
\operatorname{tr}\left[\tilde{N}^{T}\left(\hat{\Psi}-\Psi_{0}\right)\right]=\frac{1}{2}\|\tilde{\Psi}\|_{F}^{2}+\frac{1}{2}\left\|\hat{\Psi}-\Psi_{0}\right\|_{F}^{2}-\frac{1}{2}\left\|\Psi-\Psi_{0}\right\|_{F}^{2}
$$

Further

$$
\begin{aligned}
\dot{V} \leq & -\lambda_{\min }(Q)\|E\|^{2}-\lambda_{\text {min }}(\tilde{Q})\|\tilde{E}\|^{2} \\
& +2\|P \bar{b}\|(\|E\|+\|\tilde{E}\|)\left[\gamma_{1}\|\tilde{Z}\|_{F}+\gamma_{2}\right] \\
& +2 \Theta\|\tilde{E}\|\left[\alpha_{1}\|\tilde{Z}\|_{F}+\alpha_{2}\right]-\frac{k}{2}\|\tilde{Z}\|_{F}{ }^{2}+\bar{Z} .
\end{aligned}
$$

Grouping terms

$$
\begin{aligned}
\dot{V} \leq & -\lambda_{\text {min }}(Q)\left\|\left.E\right|^{2}-\lambda_{\text {min }}(\tilde{Q})\right\| \tilde{E} \|^{2} \\
& +2\|P \bar{b}\|(\|E\|+\| \tilde{E} \mid)\left[\gamma_{1}\|\tilde{Z}\|_{F}+\gamma_{2}\right] \\
& +2\|\tilde{E}\|\left[\Theta\left(\alpha_{1} \|\left.\tilde{Z}\right|_{F}+\alpha_{2}\right)+\|P \bar{b}\|\left(\gamma_{1}\|\tilde{Z}\|_{F}+\gamma_{2}\right)\right] \\
& -\frac{k}{2}\|\tilde{Z}\|_{F}^{2}+\bar{Z}
\end{aligned}
$$

and further put in the for

$$
\begin{aligned}
\dot{V} \leq & -\left.\lambda_{\text {min }}(Q)|| E\right|^{2}-\lambda_{\text {min }}(\tilde{Q})\|\tilde{E}\|^{2} \\
& +2\left\|P \overline { b } \left|\left\|E\left|\left\|\left[\alpha_{1}|| \tilde{Z} \|_{F}+\alpha_{2}\right]+2\right\| \tilde{E}\right| \mid\left[k_{1} \|\left.\tilde{Z}\right|_{F}+k_{2}\right]\right.\right.\right. \\
& -\frac{k}{2} \|\left.\tilde{Z}\right|_{F} ^{2}+\bar{Z}
\end{aligned}
$$

Upon completion of squares, we get

$$
\begin{aligned}
\dot{V} \leq- & \left(\lambda_{\min }(Q)-1\right)\|E\|^{2}+2 \gamma_{2}\|P \bar{b}|\|| E \mid \\
& -\left(\lambda_{\min }(Q)-1\right)\left\|\left.\tilde{E}\right|^{2}+2 k_{2}\right\| P \bar{b}|\|\mid \tilde{E}\| \\
& -\left[\|\tilde{E}\|-k_{1}\|\tilde{Z}\|_{F}\right]^{2}+k_{1}^{2}\|\tilde{Z}\|_{F}^{2} \\
& -\left[\|\tilde{E}\|-\gamma_{1}\left\|P \bar{b}\left|\|\mid \tilde{Z}\|_{F}\right]^{2}+\left[\gamma_{1}\|P \bar{b}\|^{2}\|\tilde{Z}\|_{F}\right]^{2}\right.\right. \\
& -\frac{k}{2}|| \tilde{Z} \|_{F}^{2}+\bar{Z} .
\end{aligned}
$$

One more completion of squares allows for the following upper bound: 


$$
\begin{aligned}
\dot{V} \leq & -\left(\lambda_{\min }(Q)-2\right)\|E\|^{2}+2 \gamma_{2}^{2}\|P \bar{b}\|^{2} \\
& -\left(\lambda_{\min }(\tilde{Q})-2\right)\|\tilde{E}\|^{2}+k_{2}^{2} \\
& -\left(\frac{k}{2}-k_{1}^{2}-\left[\gamma_{1}\|P \bar{b}\|\right]^{2}\right)\|\tilde{Z}\|_{F}^{2}+\bar{Z}
\end{aligned}
$$

Either of the following conditions:

$$
\begin{aligned}
& \|E\|>\sqrt{\frac{\|P \bar{b}\|^{2} \gamma_{2}{ }^{2}+k_{2}{ }^{2}+\bar{Z}}{\lambda_{\text {min }}(Q)-2}} \\
& \|\tilde{E}\|>\sqrt{\frac{\|P \bar{b}\|^{2} \gamma_{2}{ }^{2}+k_{2}{ }^{2}+\bar{Z}}{\lambda_{\text {min }}(\tilde{Q})-2}}
\end{aligned}
$$

and

$$
\|\tilde{Z}\|_{F}>\sqrt{\frac{\|P \bar{b}\|^{2} \gamma_{2}^{2}+k_{2}^{2}+\bar{Z}}{\frac{k}{2}-k_{1}^{2}-\left[\gamma_{1}\|P \bar{b}\|\right]^{2}}}
$$

will render $\dot{V}<0$ outside a compact set.

Define the ball in the space of the error variables

$$
B_{\gamma}=\left\{\zeta \in B_{R},\|\zeta\| \leq \gamma\right\}
$$

outside which $\dot{V}<0$. Note from (37) that $B_{\gamma} \subset B_{R}$.

Consider the Lyapunov function candidate in (39) and write it as $V=\zeta^{T} \Gamma \zeta$. Let $H_{v}$ be the maximum value of the Lyapunov function $V$ on the edge of $B_{\gamma}$.

$$
H_{v}=\max V=\gamma^{2} \Gamma_{M}
$$

Introduce the level set

$$
\Omega_{\gamma}=\left\{\zeta, V=H_{v}\right\}
$$

Let $\alpha$ be the minimum value of the Lyapunov function $V$ on the edge of $B_{R}$.

$$
\alpha=\min _{\|\zeta\|=R} V=R^{2} \Gamma_{m}
$$

Define the level set

$$
\Omega_{\alpha}=\left\{\zeta \in B_{R}, V=\alpha\right\}
$$

The condition in (37) ensures that $\Omega_{\gamma} \subset \Omega_{\alpha}$, and thus ultimate boundedness of $\zeta$.

\section{Application}

To illustrate the performance of the proposed adaptive controller in the presence of unstructured uncertainties, we consider both nonlinear systems, Van der Pol and the tunnel diode circuit example:

\subsection{Tunnel Diode Circuit Example}

Consider a tunnel diode circuit represented by the model [2].

$\left\{\begin{array}{l}\dot{x}_{1}=\frac{1}{C} x_{2}-\frac{1}{C} h\left(x_{1}\right) \\ \dot{x}_{2}=-\frac{R}{L} x_{2}-\frac{1}{L} x_{1}+\frac{u}{L}\end{array}\right.$

where the output $y=x_{1}$ has a full relative degree $\left(r_{l}=n_{1}=2\right)$. Noting that $x_{1}$ be the voltage across the capacitor $C$ and $x_{2}$ is the current through the inductor $L$. The initial conditions were set as $x_{1}(0)=0.1, x_{2}(0)=0.0005$ and the element values of the circuit are $R=1.5 \mathrm{k} \Omega, L=1 \mathrm{nH}$, $C=2 \mathrm{pF}$.

The function $h: \Re \rightarrow \Re$ represents the nonlinear characteristic of the tunnel diode [2]

$$
h\left(x_{1}\right)=x_{1}+2 x_{1}^{2}+x_{1}^{3}-x_{1}^{4}-x_{1}^{5}
$$

The output $y$ has a full relative degree: $n_{1}=r_{1}=2$.

\subsection{Van der Pol Example}

The VDP nonlinear system is described as follow [19].

$\left\{\begin{array}{l}\dot{x}_{1}=x_{2} \\ \dot{x}_{2}=-x_{1}+2 \cdot x_{2}-2 x_{1}^{2} x_{2}+\frac{u}{\sqrt{|u|+0.1}}\end{array}\right.$

with initial conditions $x_{1}(0)=0.5, x_{2}(0)=1.5$. The output $y=x_{1}$ has full relative degree: $n_{2}=r_{2}=2$.

The following dynamic compensator:

$\left\{\begin{array}{l}\dot{\eta}=-5.1 \eta+5 \tilde{y} \\ v_{d c}=-6.3 \eta+8.2 \tilde{y}\end{array}\right.$

places the poles of the closed-loop error dynamics in (10) of both nonlinear systems at $-3.1,-1.05 \pm j$ ( refer to [4] for more details).

The observer dynamics in (17) were designed so that its poles are five times faster than those of the error dynamics. We implemented six neurons in the hidden layer, and the following sigmoidal basis function:

$\sigma(x)=\frac{1}{1+e^{-a x}}$ 
with $a=1$. The adaptation gains were set to $F=2 . I$, with sigma modification gain $k=0.73$.

The contribution of this paper is to design an adaptive output feedback control methodology using only one single hidden layer (SHL NN) that cancel the nonlinearities of both nonlinear systems, Van der Pol and tunnel diode circuit model, what bring to force the system measurement to track reference trajectory with bounded errors.

\section{Simulation Results}

First, setting the output $y=x_{1}$ for the highly uncertain nonlinear systems, tunnel diode circuit and Van der Pol example. However, the reader is reminded that the controller has been designed given only the fact that we have full relative degree $r=2$. In the proposed control scheme, a fixed structure dynamic compensator is designed to stabilize the linearized system [2]. Then, we employ feedback linearization, coupled with an on-line $\mathrm{NN}$ to compensate adaptively for modelling errors. A signal, comprised of a linear combination of the measured tracking error and the compensator states, is used to adapt the $\mathrm{NN}$ weights [4].

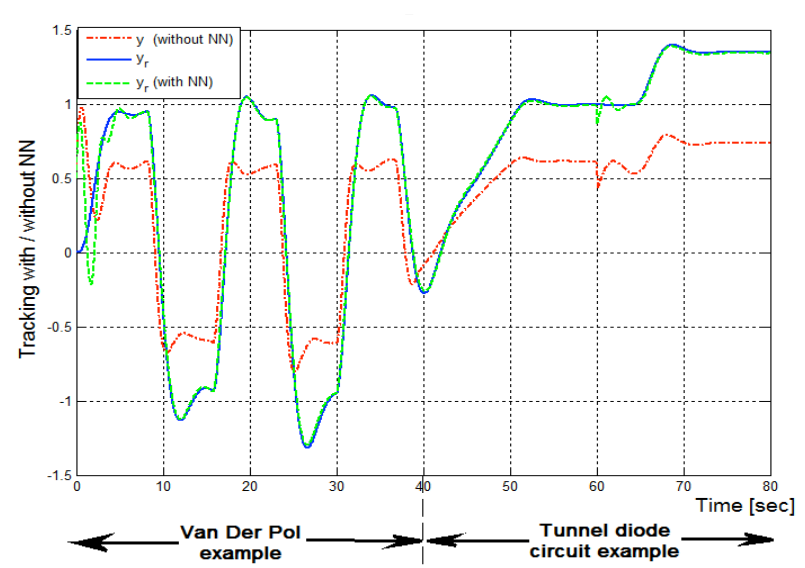

Fig. 1. Tracking without NN (red line) and with NN (green line).

Fig.1. compares the system response without NN augmentation (red line) with the reference model output (blue line), clearly demonstrating the almost unstable oscillatory behavior caused by the nonlinear elements in the Van der Pol model in the first half time [0-40] seconds and the nonlinearities of the tunnel diode equation in the last half time [40-80]s. Also, we validate that with $\mathrm{NN}$ augmentation (green line), these oscillations are eliminated after a period of about 2 seconds. This is accounted for the successful identification of the model inversion error (solid line) by the output of SHL NN (dashed line), which is also illustrated in Fig.2.

Fig. 3 compares the control efforts without and with adaptation, where the NN based adaptive controller exhibits a steady state tracking error. This error can be minimized when designing an excellent linear compensator.

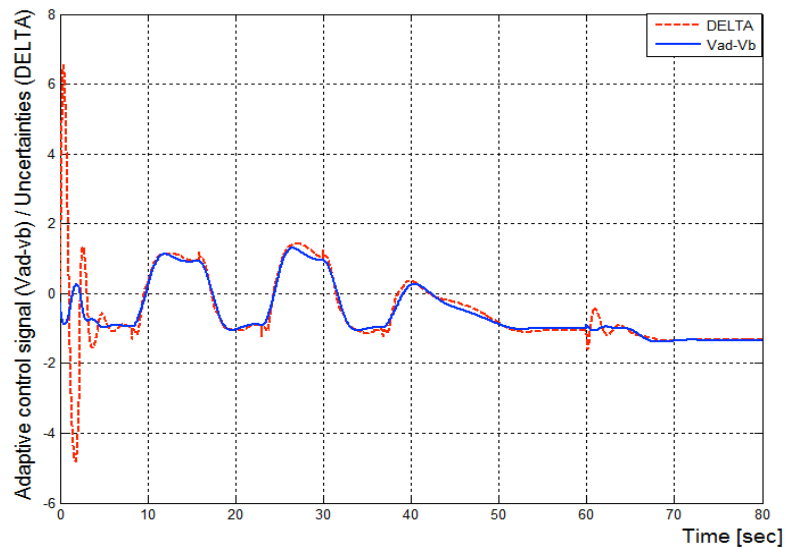

Fig. 2. Comparison between adaptive control signal $\left(u_{a d}\right)$ and the computed uncertainties $(\Delta)$.

Moreover, one chooses a good structure of the neural network i.e. number of iterations and the number of neurones in the hidden layer, in order to avoid the phenomenon of on-training. The NN controller weights history is clearly shown in Fig.4.

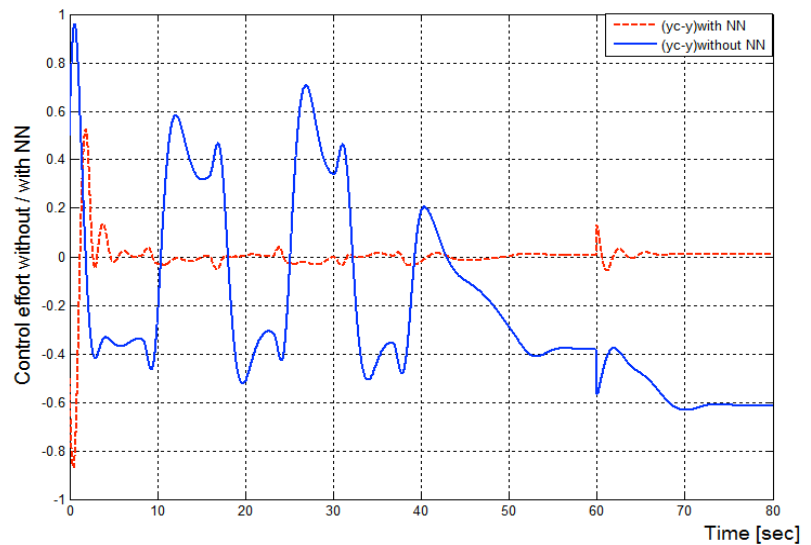

Fig. 3. Control effort with / without NN.

\subsection{Test of Robustness of the Adaptive Output Feedback Control}

To illustrate that the proposed approach is applicable to systems with parametric uncertainty and unmodeled dynamics, the rate of nonlinearities $(\Delta)$ will be increased $(50 \%$ and $100 \%)$ of its norm value. Once again, our perspective is to validate the effectiveness of the proposed $\mathrm{NN}$ with only one single hidden layer that compensate for highly unstructured uncertainties.

Fig.5 confirms that the adaptive feedback control augmented using only single hidden layer neural networks overcomes the effect of nonlinearities on the tracking accuracy. This is justified by the good identification of nonlinearities $(\Delta)$ by the $\mathrm{NN}$ which is also illustrated in Fig.2. 


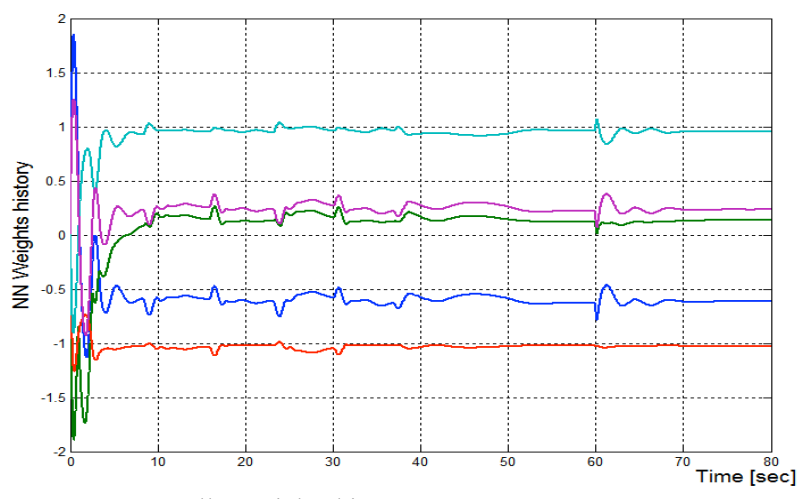

Fig. 4. NN controller weights history.

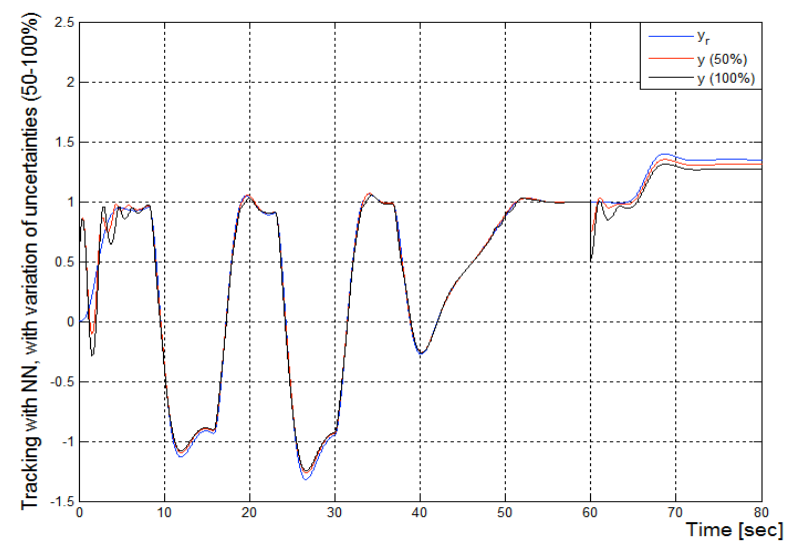

Fig. 5. Tracking with $\mathrm{NN}$, with increasing nonlinearities $(50 \%$ and $100 \%$ ) of its norm value $(\Delta)$.

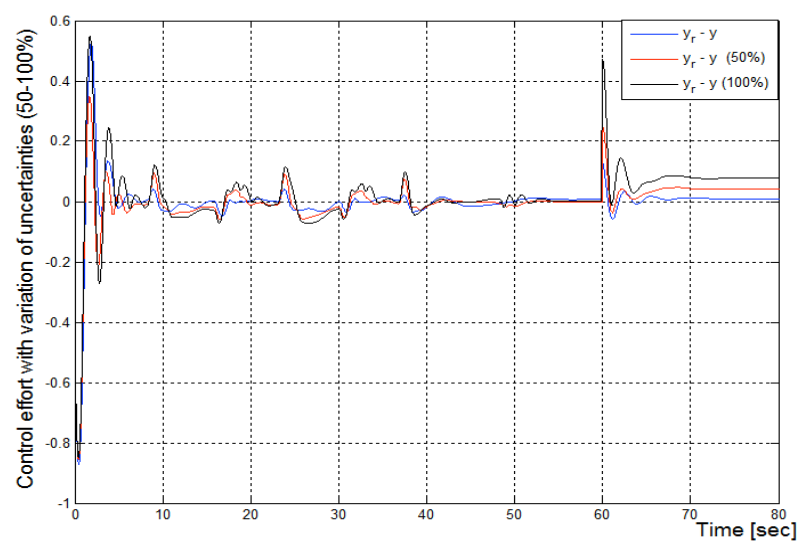

Fig. 6. Control effort (with $\mathrm{NN}$ ) with variation of uncertainties (50 and $100 \%)$.
The NN augmentation adjusts $\left(u_{a d}\right)$ for nonlinear effects $(\Delta)$ and this has allowed us to minimize the tracking errors $\left(y_{r}-y\right)$, such as illustrated in fig.6. As expected, the SHL NN improves the tracking performance due to its ability to "model" nonlinearities on-line, even with variations of $(\Delta)(50$ and $100 \%)$, such as presented in fig.7.

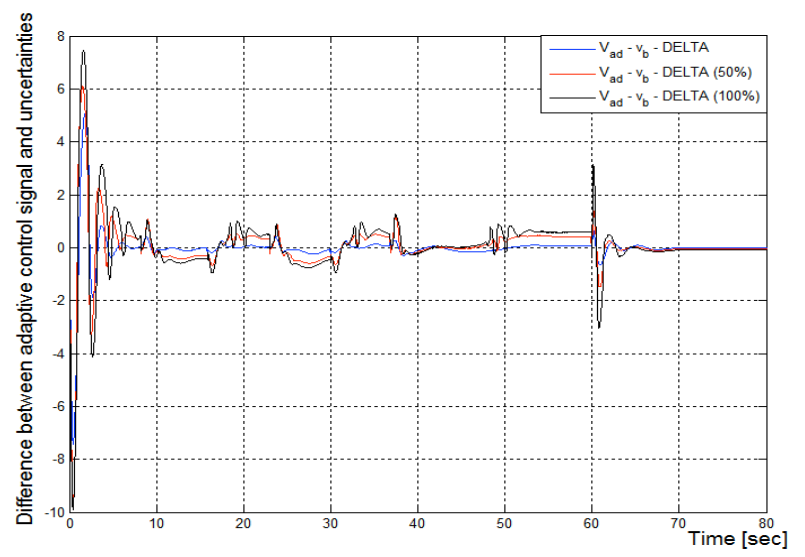

Fig. 7. Difference between adaptive control $\left(u_{a d}\right)$ signal and the uncertainties varied $(\Delta)$.

\section{Conclusion}

A new approach has been proposed for adaptive output feedback control of uncertain nonlinear systems in the presence of both parametric uncertainty and unmodeled dynamics. The obtained controller is then augmented by only one SHL NN used to compensate adaptively for the neglected terms and unstructured uncertainties. A simple linear observer was introduced to estimate the derivatives of the tracking error. These estimates are used as inputs to the neural network and in the adaptation laws as an error signal. Ultimate boundedness of all error signals was proven by Lyapunov's direct method. The methodology is applicable to nonlinear systems of unknown but bounded dimension, as long as the relative degree is known. Through computer simulation, we were able to demonstrate that the proposed intelligent adaptive output feedback controller provides strong robustness to modelling inaccuracies, ability to handle both complicated nonlinearity and arbitrary complexity, and excellent tracking performance was succeeded.

\section{References}

1. J.H. Ahrens and H.K. Khalil, Output feedback control using high-gain observers in the presence of measurement noise, In Proc. of the American Control Conference, vol. 5, pp. 4114 4119, Boston, MA, USA (2004).

2. H.A. Abbas, M. Belkheiri, and B. Zegnini, Feedback linearization control of a class of nonlinear uncertain systems using neural networks, International Journal on Advanced Electrical Engineering, vol. 1(2), pp. 121-131 (2013).

3. F. Angulo, M.D. Bernardo, U. Montanaro, A. Rincon, and S. Santini, Adaptive control for state dependent switched systems in Brunovsky form, In 2013 European Control Conference (ECC), Zürich, Switzerland (2013).

4. M. Belkhiri, H.A. Abbas, and B. Zegnini, Feedback linearization control of nonlinear uncertain systems using single hidden layer neural networks, In 11th International Multi-Conference on Systems, Signals \& Devices (SSD), pp. 1-6 (2014).
5. M. Belkheiri and F. Boudjema, Neural network augmented backstepping control for an induction machine, Int. J. Modelling, Identification and Control, vol. 5(4), pp.288-296 (2008).

6. J. Brasch and J. Pearson, Pole placement using dynamic compensators, IEEE Trans. Automat. Contr., vol. AC-15, pp. 3443 (1970).

7. A.J. Calise, S. Lee, and M. Sharma, Development of a reconfigurable flight control law for a tailless aircraft, J. Guidance, Contr., Dynamics, vol. 24(5), pp. 896-902 (2001).

8. Y.C. Chang, Adaptive fuzzy-based tracking control for nonlinear SISO systems via VSS and approaches, IEEE Trans. Fuzzy Syst., vol. 9(2), pp. 278-292 (2001).

9. D. Chen, G. Zhou, Y. Liu, and G. Lei, Neural learning control of general Brunovsky systems with a novel exponential stability theorem, In International Conference on Information Science and Technology, Nanjing, Jiangsu, China (2011). 
10. J.Y. Choi and J. Farrell, Observer-based backstepping control using on-line approximation, In Proc. Amer. Control Conf., Chicago, IL, pp. 3646-3650 (2000).

11. J.M. Daly and H.M. Schwartz, Non-linear adaptive output feedback control of robot manipulators, In Proc. of the 2005 IEEE International Conference on Robotics and Automation, pp. 1687-1693 (2005)

12. S. Dingxin, X. Yunxiang, and W. Xiaogang, The research of input-output linearization and stabilization analysis of internal dynamics on the CCM boost converter, In Proc. IEEE ICEMS 2008, pp. 1860-1864 (2008).

13. J.A. Farrell, M. Polycarpou, M. Sharma, and W. Dong, Command filtered backstepping, IEEE Trans. Autom. Control, vol. 54(6), pp. 1391-1395 (2009).

14. N. Hovakimyan, R.T. Rysdyk, and A.J. Calise, Dynamic neural networks for output feedback control, In Proc. Conf. Decision Contr., pp. 1685-1690 (1999).

15. W. Gao and R.R. Selmic, Neural network control of a class of nonlinear systems with actuator saturation, IEEE Transactions on Neural Networks, vol. 17(1) (2006).

16. S.S. Ge, C.C. Hang, and T. Zhang, Adaptive neural network control of nonlinear systems by state and output feedback, IEEE Trans. Syst., Man, Cybern. B, Cybern., vol. 29(6), pp. 818-828 (1999).

17. S.S. Ge and C. Wang, Adaptive neural control of uncertain MIMO nonlinear systems, IEEE Trans. Neural Netw., vol. 15(3), pp. 674-692 (2004).

18. Y. Guo and P.Y. Woo, An adaptive fuzzy sliding mode controller for robotic manipulators, IEEE Trans. Syst., Man, Cybern. A, Syst. Humans, vol. 33(2), pp. 149-159 (2003).

19. N. Hovakimyan, F. Nardi, and A.J. Calise, A Novel error observer-based adaptive output feedback approach for control of uncertain systems, IEEE Transactions on Automatic Control, vol. 47(8), pp. 1310-1314 (2002).

20. Z.P. Jiang, A combined backstepping and small-gain approach to adaptive output feedback control, Automatica, vol. 35(6), pp. 1131-1139 (1999)

21. Y. Kim and F.L. Lewis, High level feedback control with neural networks, World Scientific, Singapore (1998).

22. M. Kristic, I. Kanellakopoulos, and P. Kokotovic, Nonlinear and adaptive control design, Wiley, New York (1995).

23. Y.G. Leu, T.T. Lee, and W.Y. Wang, Observer-based adaptive fuzzy-neural control for unknown nonlinear dynamical systems, IEEE Trans. Syst., Man, Cybern. B, Cybern., vol. 29(5). pp. 583591 (1999).

24. F.L. Lewis, A. Yesildirek, and K. Liu, Multilayer neural-net robot controller with guaranteed tracking performance, IEEE Trans. Neural Networks, vol. 7(2), pp. 1-12 (1996).
25. J.D. Lindlau and C.R. Knospe, Feedback Linearization of an Active Magnetic Bearing With Voltage Control, IEEE Transactions on Control Systems Technology, vol. 10(1) (2002).

26. V. Nekoukar and A. Erfanian, Adaptive fuzzy terminal sliding mode control for a class of MIMO uncertain nonlinear systems, Fuzzy Sets Syst., vol. 179(1), pp. 34-49 (2011).

27. M.M. Polycarpou, Stable adaptive neural control scheme for nonlinear systems, IEEE Trans. Automat. Contr., vol. 41(3), pp. 447-451 (1996).

28. A. Praly and Z.P. Jiang, Stabilization by output feedback for systems with ISS inverse dynamics, Syst. Control Lett., vol. 21, pp. 19-33 (1993).

29. S. Seshagiri and H.K. Khalil, Output feedback control of nonlinear systems using RBF neural networks, IEEE Trans. Neural Networks, vol. 11(1), pp. 69-79 (2000).

30. J. Shin, H.J. Kim, and Y. Kim, Adaptive feedback linearization for an uncertain nonlinear system using support vector regression, In American Control Conference Marriott Waterfront, Baltimore, MD, USA (2010).

31. S. Tong and H.X. Li, Fuzzy adaptive sliding-mode control for MIMO nonlinear systems, IEEE Trans. Fuzzy Syst., vol. 11(3), pp. 354-360 (2003).

32. S. Tong and Y. Li, Observer-based fuzzy adaptive control for strict-feedback nonlinear systems, Fuzzy Sets Syst., vol. 160(12), pp. 1749-1764 (2009)

33. C.S. Tseng and B.S. Chen, Multi-objective PID control design in uncertain robotic systems using neural network elimination scheme, IEEE Trans. Syst., Man, Cybern. A, Syst. Humans, vol. 31(6), pp. 632-644 (2001).

34. C.H. Wang, H.L. Liu, and T.S. Lin, Direct adaptive fuzzy-neural control with state observer and supervisory controller for unknown nonlinear dynamical systems, IEEE Trans. Fuzzy Syst., vol. 10(1), pp. 39-49, (2002).

35. M. Wang, B. Chen, and P. Shi, Adaptive neural control for a class of perturbed strict-feedback nonlinear time-delay systems, IEEE Trans. Syst., Man, Cybern. B, Cybern., vol. 38(3), pp. 721730 (2008).

36. Z. Wu, X. Xie, P. Shi, and Y. Xia, Backstepping controller design for a class of stochastic nonlinear systems with Markovian switching, Automatica, vol. 45(4), pp. 997-1004 (2009).

37. Y. Yang, G. Feng, and J. Ren, A combined backstepping and small-gain approach to robust adaptive fuzzy control for strictfeedback nonlinear systems, IEEE Trans. Syst.,Man, Cybern. A, Syst. Humans, vol. 34(3), pp. 406-420 (2004).

38. Y. Yang and C. Zhou, Adaptive fuzzy $\mathrm{H} \infty$ stabilization for strictfeedback canonical nonlinear systems via backstepping and small-gain approach, IEEE Trans. Fuzzy Syst., vol. 13(1), pp. 104-114 (2005). 